\title{
New Approach for Codend Selectivity: a Case Study of Coastal Beach Seine for Big-Scale Sand Smelt (Atherina boyeri) Fishery in İznik Lake
}

\author{
Mehmet CiLBiz ${ }^{1, *}$ (D), Rahmi UYSAL ${ }^{1}$, Zafer TOSUNOĞLU², Celalettin AYDIN ${ }^{2}$, Ahmet \\ $\mathrm{ALP}^{3}$, Fuat Bígín ${ }^{1}$
}

${ }^{1}$ Fisheries Research Institute,32500, Eğirdir-Isparta.

${ }^{2}$ Ege University Faculty of Fisheries, 35100, Bornova-İzmir.

${ }^{3}$ Kahramanmaraş Sütçü İmam University, Agricultural Faculty, Fisheries Department, Kahramanmaraş.

\section{Article History}

Received May 21, 2019

Accepted Jun 15, 2020

First Online Jun 18, 2020

\section{Corresponding Author}

Tel.: +902463133460

E-mail:mehmetcilbiz@gmail.com

\section{Keywords}

Inland fisheries

Artisanal fisheries

Sustainable fishery

GAMs

\begin{abstract}
Sand smelt (Atherina boyeri Risso, 1810), which is an exotic species with invasive character, is the third most caught species in the small-scale fisheries among the inland fisheries in Turkey. The fact that it has become an important source of income for the fishermen with low income due to its export potential has led to the maintenance of its stock recently. In this study, the selectivity of coastal beach seine used in the fishing of the species was investigated. A specially designed coastal beach seine with $10 \mathrm{~m}$ codend consisting of 4 different compartments was used in the study. Among the compartments of the codend, which was surrounded by a $6 \mathrm{~mm}$ mesh cover, in the codend panels mesh with 9, 12 and $14 \mathrm{~mm}$ were used. Field studies were carried out on a monthly basis in 4 different stations of İnik Lake in 2014. Selectivity data were collected by the covered codend method and analyzed by means of a logistic equation with the maximum likelihood method. A totally 3053 individuals were caught length ranged from $2.6-11.2 \mathrm{~cm}$ and weight ranged from $0.11-11.02 \mathrm{~g}$. The mean $L_{50}$ values of 14, 12- and 9-mm panels were determined as $6.71,5.62$ and $4.51 \mathrm{~cm}$ total length, respectively. It is concluded that $12 \mathrm{~mm}$ mesh size panel is suitable for sustainable $A$. boyeri fishery by beach seine net when considered given by length at first maturity size.
\end{abstract}

\section{Introduction}

Actually, marine origin species big scale sand smelt (Atherina boyeri Risso, 1810) has rapid spread to isolated inland water thanks to adaptability. It was first reported in 1940 from Sapanca Lake in Turkey (Battalgil, 1941; Ekmekçi et al., 2013). After 1988, when first observation year in Iznik Lake, $A$. boyeri is one of the first commercial fish species of the lake and its production has shown increase trend year by year (Dogan, 2009). Production was started from 60 t/year in 1989 (Dogan, 2009), and increasing approximately $1350 \mathrm{t}$ in 2017.

During the $A$. boyeri adaptation periods to the lake ecosystem fishermen were investigated the suitable fishing methods and gears. In the first years, fishermen used small beach seine (maximum length approximately 50-60 m) with manpower. Following years, gears are developed with the giant ones (length range 200-400 m) and also engine power began to be used instead of manpower. Portable net drum (with tractor) has been utilized for hauling of very big seine from the lake. In 2007, annual gross income of a fisherman was reported as 27788 \$USD based on $A$. boyeri fishing in İnik Lake (Dogan, 2009).

According to the 2017 data, A. boyeri was the third caught species in Turkey inland fisheries. Their economic value of $4892 \mathrm{t}$ of $A$. boyeri production was almost 2065150 \$USD (TUIK, 2019). 1/4 of total Turkey's production has been provided from İznik Lake. Almost all individuals are proceeding as a crisps and export to 
some European county such as Italy, England, France and Spain. Due to hardening of scale and spine of larger individuals (larger than 6-7 cm total length) not preferred for crisps production, this circumstance is causes abnormal fishing pressure on young individuals.

Iznik Lake is the fifth larger lake of Turkey with 300 $\mathrm{km}^{2}$ surface area. A total 180 licensed fishermen work as commercially in four fishing area. Besides A. boyeri, Cyprinus carpio, Carassius gibelio, Rutilus frisii, Siluris glanis and Astacus leptodactylus have been caught in the lake. Fishing operation for sand smelt is made by beach seine with 4-5 fishers (a team). According to the 2018 data, there are 42 teams in the lake. Beach seines with smaller than $9 \mathrm{~mm}$ mesh size (stretched) were commonly used for $A$. boyeri fishing.

There are some studies conducted on $A$. boyeri fishing; Rodríguez-Climent et al. (2012) presented gillnet selectivity of $A$. boyeri in Ebro Delta Lagoon (North-East, Spain) by using SELECT method. Catch composition, CPUE, bycatch and discard rate studies were carried out on beach seine fishing (Broadhurst et al., 2008; Akyol, 2003; Cabral et al., 2003; El-Mor et al., 2002; Gray \& Kennelly, 2003; Vanderklift et al., 1998; Hutchings \& Lamberth, 2002a; Hutchings \& Lamberth, 2002b). However, there are very limited beach seine selectivity studies both in the sea and inland water; Tosunoğlu (2003), determined Mullus barbatus, Pagellus erythrinus and Diplodus annularis selectivity parameters in Turkey's Aegean Sea by using covered codend method. The other study was conducted by Tesfaye, Wolff and Taylor. (2016) for investigated O. niloticus, C. gariepinus and $C$. carpio selectivities in Lake Koka (central Ethiopia).

Brčić et al. (2018) investigated the selectivity of the commercial creels with $41 \mathrm{~mm}$ mesh, which were used in fishing Nephrops norvegicus in the Mediterranean, using the control creel with $41 \mathrm{~mm}$ mesh and he found the 150 value as 31.82 for 1 soak time. In another study, Deval et al. (2016) investigated the selectivity of TO and T9O codends with $50 \mathrm{~mm}$ mesh on commercial shrimp species in Turkish deepwater trawl fishery, Eastern Mediterranean; it was found that there was an increase in the escape rates of Aristaeomorpha foliacea, Aristeus antennatus, Parapenaeus longirostris and Plesionika martia in case the codend was transformed from TO to T90. Sala, Brčić, Herrmann, Lucchetti, \& Virgili (2016) investigated the selectivity of a grid system, which was designed for grading of striped venus clam (Chamelea gallina) after fishing with hydraulic dredge. The grid system with 5 different sequential holes spacing (Filter: $32.5 \mathrm{~mm}$, Grid 1: $21.5 \mathrm{~mm}$, Grid 2: $21.1 \mathrm{~mm}$ and Grid 3: $20.3 \mathrm{~mm}$ and Filter: $10.5 \mathrm{~mm}$ ) was used in the study. $L_{50}$ sizes for Grid 1, Grid 2 and Grid 3 were found to be 25.01, 24.29 and $22.84 \mathrm{~mm}$. A similar approach to our study was demonstrated in pooling the data used in the calculations. However, there are not any study conducted on beach seine selectivity for $A$. boyeri fishing.

In this study, our goal is to determine the selectivity characteristics of coastal beach seine, which was designed to determine the selectivity of codends with different meshes $(9,12-$ and $14-\mathrm{mm}$ nominal stretched mesh size) in the same hauling simultaneously, in sand smelt fishing.

\section{Material and Method}

\section{Study Area}

The study was conducted in İnik Lake that is one of most important $A$. boyeri producer (Figure 1). As a tectonic origin, Lake İznik locate southeastern part of the Marmara region in Turkey. Altitude is $85 \mathrm{~m}$ and maximum depths was determined as a little bit more than $70 \mathrm{~m}$ at near south coast. Lake's surface area is $\mathbf{2 8 5}$ $\mathrm{km}^{2}$ and length is $32 \mathrm{~km}$ (Ceribasi, 2018). Lake located between $40^{\circ} 23^{\prime}-40^{\circ} 30^{\prime} \mathrm{N}$ latitudes and $29^{\circ} 20^{\prime}-29^{\circ} 42^{\prime} \mathrm{E}$ longitudes (Yağci \& Ustaoğlu, 2012).

\section{Sampling Process}

Experiments were carried out 4 different station of lake in 2014 with monthly period. Only one hauling

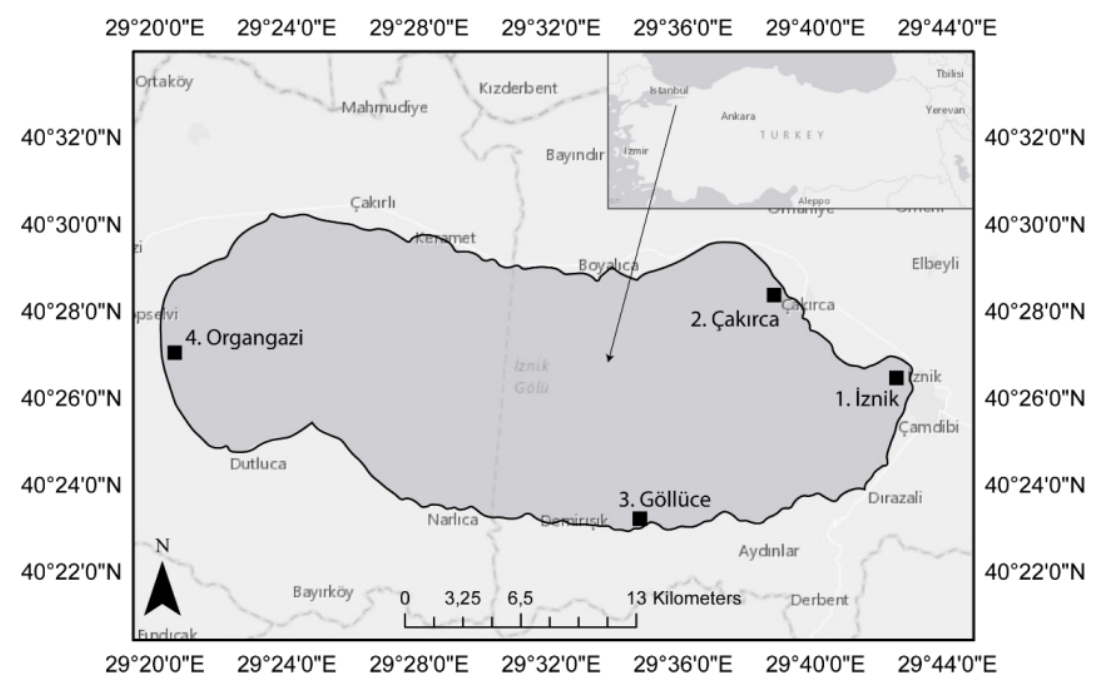

Figure 1. Study area and sampling stations. 
applied each station. A beach sine used in hauling which was specially designed and rigged to determine multi codend mesh selectivity parameters at same hauling operation. More information about the geometry and characteristics of the used beach seine given Figure 2 .

Multimesh codend model was generated from four different codend compartment by connected with each other using touch \& close tape. First compartment's tagend rigged codend mesh with $14 \mathrm{~mm}$ and it surrounded with cover mesh $(6 \mathrm{~mm})$; second compartment's tagend rigged codend mesh with $12 \mathrm{~mm}$ and it surrounded with cover mesh $(6 \mathrm{~mm})$; third compartment's tag-end rigged codend mesh with $9 \mathrm{~mm}$ and it surrounded with cover mesh ( $6 \mathrm{~mm}$ ) and finally all of last compartment covered by $6 \mathrm{~mm}$ cover mesh (Figure 3 ).

A unique data pooled model was used in the study due to the possibility that the length distributions of the haul obtained from different stations in the same period could be different. Due to the possibility that the schools of different length distributions will be evaluated each time during the sampling, it was expected to see that there were inconsistencies in the estimated $L_{50}$ values at the classic single codend \& cover application, which stemmed from the diverse size distributions of the schools. For this reason, a model, where the size selectivities of different codends for each school could be tried simultaneously, was necessary. Within this framework, a beach seine, on which the part that remained in the front part of each panel was taken as the codend and the back parts were taken as the cover, was used in the study, displayed in detail in Figure 3.

\section{Data Analysis}

Caught fish were classified by codend type and measured total length with $0.1 \mathrm{~cm}$ precision and gaged total weight with $0.01 \mathrm{~g}$ precision. Covered codend techniques was utilized estimation of selectivity parameters (Wileman et al., 1996). The data were analyzed using a logistic equation with the maximum likelihood method (Wileman et al., 1996). The mean selectivity of the individual hauls was calculated by taking into account between-haul variation according to Fryer (1991) using the ECModeller software (ConStat, 1995) which adopts the REML method (residual maximum likelihood). The choice of the model which best describes the data was based on the lowest value for Akaike's Information Criterion-AIC (Fryer \&

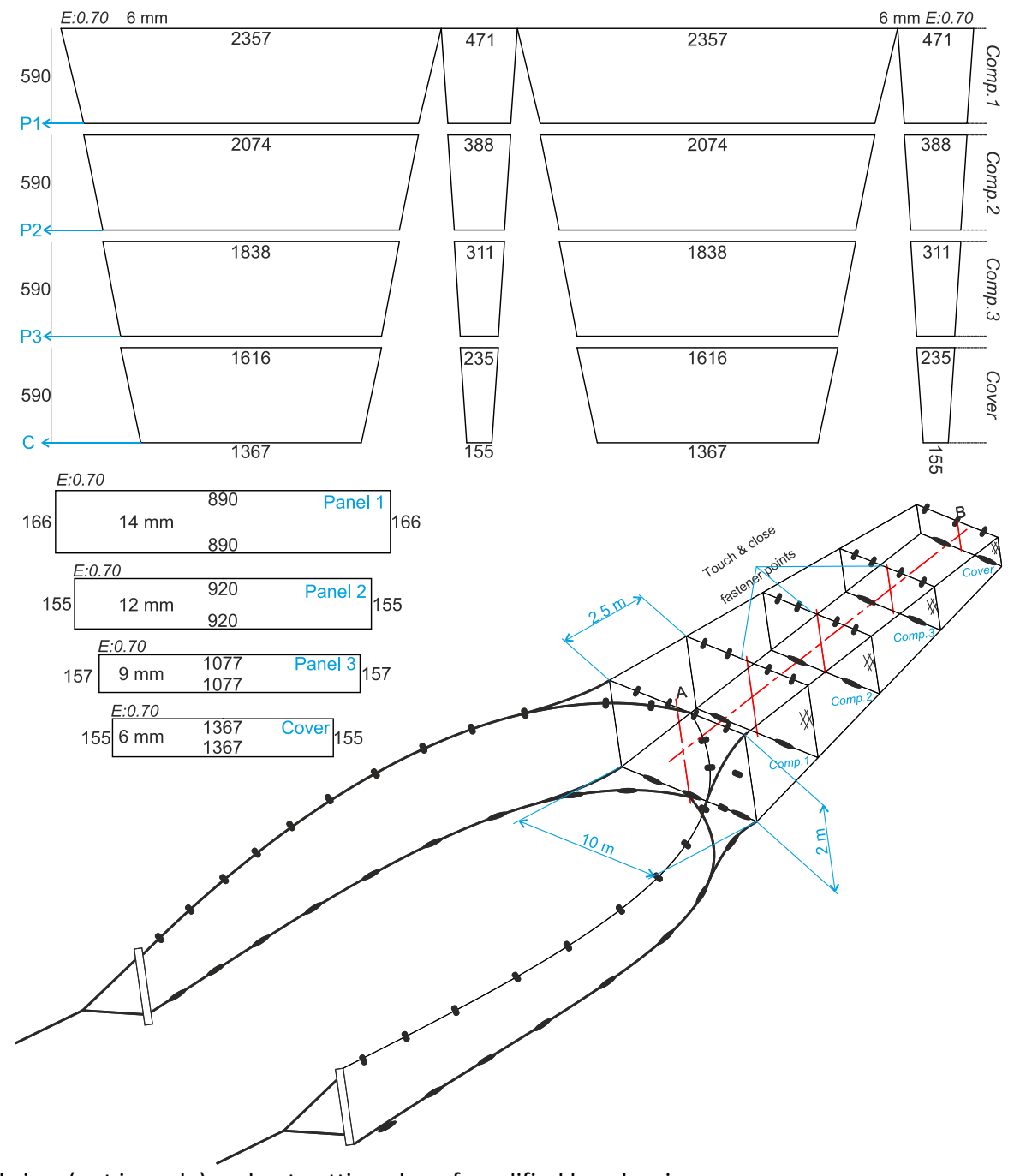

Figure 2. General view (not in scale) and net cutting plan of modified beach seine. 


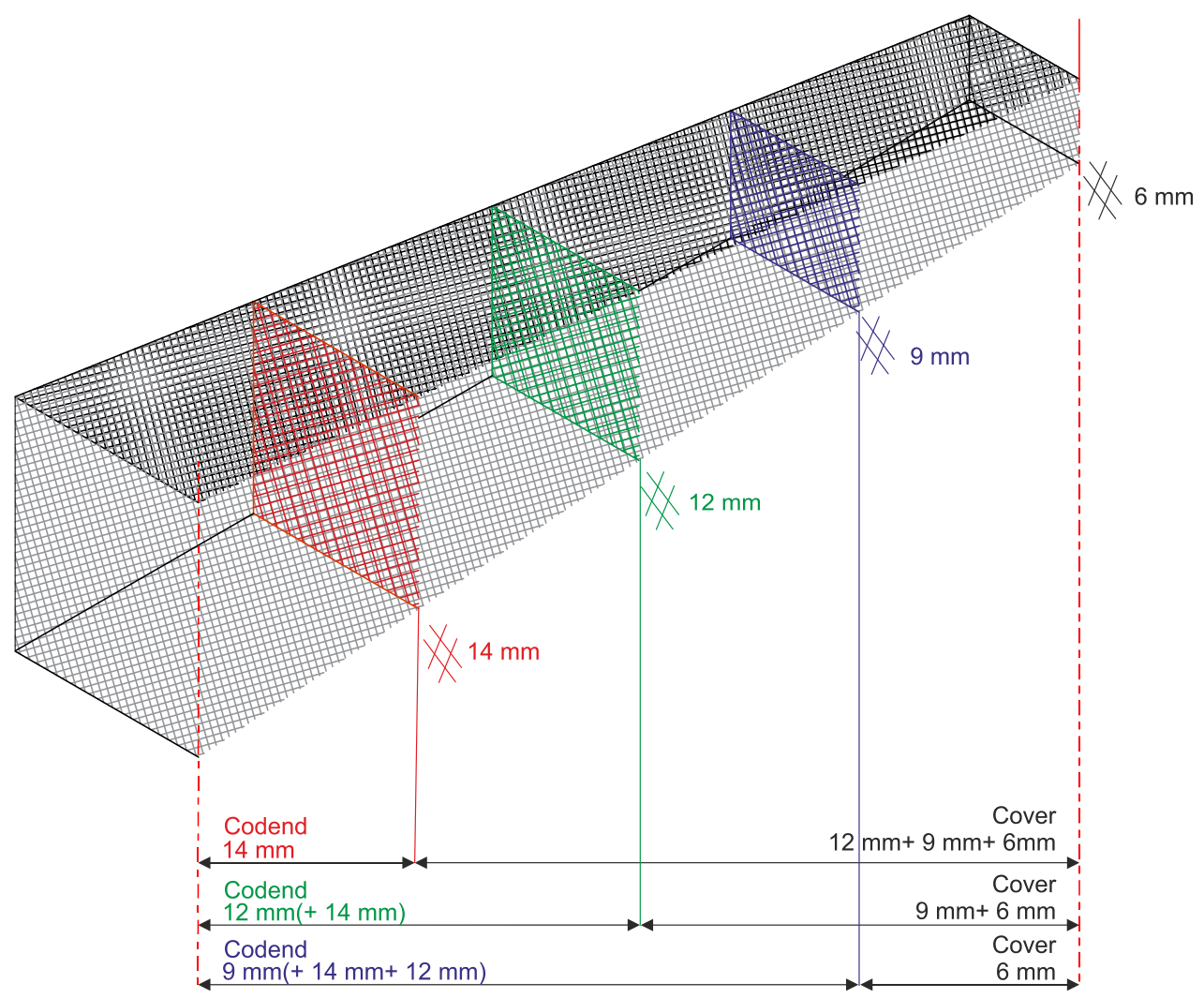

Figure 3. Data pooling process for estimating the multimesh codend selectivity (A-B sectioning which shown in Figure 2 ).

Shepherd, 1996). t-test and one-way ANOVA with Tukey ${ }^{H S D}$ were used for statistical comparing of normal disturbed data evaluations process for catch total length and weight by month. Also, Fulton's condition factor $(K)$ was calculated using $K=\left(W / T L^{3}\right) \times 100$ formula. Kolmogorov-Smirnov $(K-S)$ test was used to determine differences between size frequency distributions of fish caught by different codend. We used the "mgcv" package (Wood, 2019) in RStudio (v11.2.5019) to model the relationships between total length and influencing factors (sampling month, sampling station, codend type) in Generalized Additive Models (GAMs) evaluating process. The GAMs is given by:

$$
\text { Model: } t I \sim s(s m)+s(s s)+s(t c)
$$

Where, tl: total length, sm: sampling month, ss: sampling station, $c t$ : test codend, $s($.$) is a spline$ smoothing function. RStudio (v11.2.5019) computer software was used all statistical analysis.

\section{Result}

\section{Catch Composition}

A total of 13 valid/successful hauls were carried out for estimating selectivity parameters. End of fishing trial, a total of 3053 A. boyeri were caught total length range between $2.6-11.2 \mathrm{~cm}$ and total weight between 0.11-11.02 g. Mean total length and total weight are shown statistical differences by sampling month (Table 1) $(P<0.05)$.
Statistical differences were found mean total length and weight values $(\mathrm{P}<0.05)$ between codends. There are no statistical differences in 6 and $12 \mathrm{~mm}$ codends between male and female total length of $A$. boyeri $(\mathrm{P}>0.05)$, while differences were found in 9 and $14 \mathrm{~mm}$ codends between male and female individuals $(P<0.05)$. The highest mean total length was observed in Gölüce station, while lowest was İznik station. Statistical differences were found between mean total lengths of sampling stations $(P<0.05)$. Mean total lengths of catch are shown statistical differences between stations for same sampling period $(P<0.05)$

Used GAMs model parameters to compare catch total length and other response (sampling, month, sampling station, test codend) given Table 2 and scatter plot matrix for predictors given Figure 4. Model residual d.f. and residual deviation were found as 3042.0 and 1480.6 respectively. All response shown statistical difference by fish total lengths.

The effects of sampling month, sampling station and test codend variables on total length was modeled through GAMs and the results were given in Figure 5. It can be stated that the sampling from Iznik station negatively affected the total length, the total length as affected negatively from the summer months towards the winter months, and that the increase of mesh size used in codend affected total length positively.

From the $K-S$ test results (Table 3 ), in the all paired compare, statistical differences were found length frequency distribution of $A$. boyeri from different codend. 
Table 1. Length-weight distribution and condition factor of captured A. boyeri by sampling month

\begin{tabular}{|c|c|c|c|c|c|c|c|c|c|}
\hline \multirow{2}{*}{ Month } & \multirow{2}{*}{$N$} & \multirow{2}{*}{$(\%)$} & \multicolumn{3}{|c|}{ Total Length $(\mathrm{cm})$} & \multicolumn{3}{|c|}{ Total Weight (g) } & \multirow[t]{2}{*}{$K$} \\
\hline & & & Mean $\pm S E$ & Min. & Max. & Mean $\pm S E$ & Min. & Max. & \\
\hline March & 165 & 5.4 & $5.95 \pm 0.09^{c}$ & 3.4 & 10.3 & $1.44 \pm 0.09^{b c}$ & 0.26 & 8.24 & 0.584 \\
\hline April & 642 & 21.0 & $6.33 \pm 0.06^{d}$ & 3.1 & 11.2 & $2.14 \pm 0.07^{d}$ & 0.14 & 11.02 & 0.679 \\
\hline May & 502 & 16.4 & $6.00 \pm 0.06^{c}$ & 3.5 & 9.8 & $1.58 \pm 0.05^{c}$ & 0.19 & 6.74 & 0.623 \\
\hline June & 431 & 14.1 & $5.86 \pm 0.10^{c}$ & 2.6 & 10.6 & $1.87 \pm 0.09^{d}$ & 0.11 & 9.25 & 0.660 \\
\hline July & 573 & 18.8 & $5.16 \pm 0.05^{a}$ & 2.6 & 9.7 & $1.11 \pm 0.03^{a}$ & 0.12 & 5.65 & 0.713 \\
\hline August & 400 & 13.1 & $5.51 \pm 0.07^{b}$ & 2.8 & 9.5 & $1.42 \pm 0.05^{b c}$ & 0.22 & 4.53 & 0.738 \\
\hline September & 340 & 11.1 & $5.32 \pm 0.07^{\mathrm{ab}}$ & 3.1 & 8.9 & $1.23 \pm 0.05^{\mathrm{ab}}$ & 0.20 & 4.86 & 0.699 \\
\hline
\end{tabular}

Different letters within columns show significant differences between month $(\mathrm{P}<0.05)$.

Table 2. Comparison of models for total length using ANOVA nonparametric effects

\begin{tabular}{llll}
\hline Response & df & $F$ & $p$ \\
\hline Sampling month & 3 & 66.670 & \\
Sampling station & 2 & 42.448 \\
Test codend & 2 & 108.082 & $\mathrm{P}<0.001$ \\
\hline
\end{tabular}

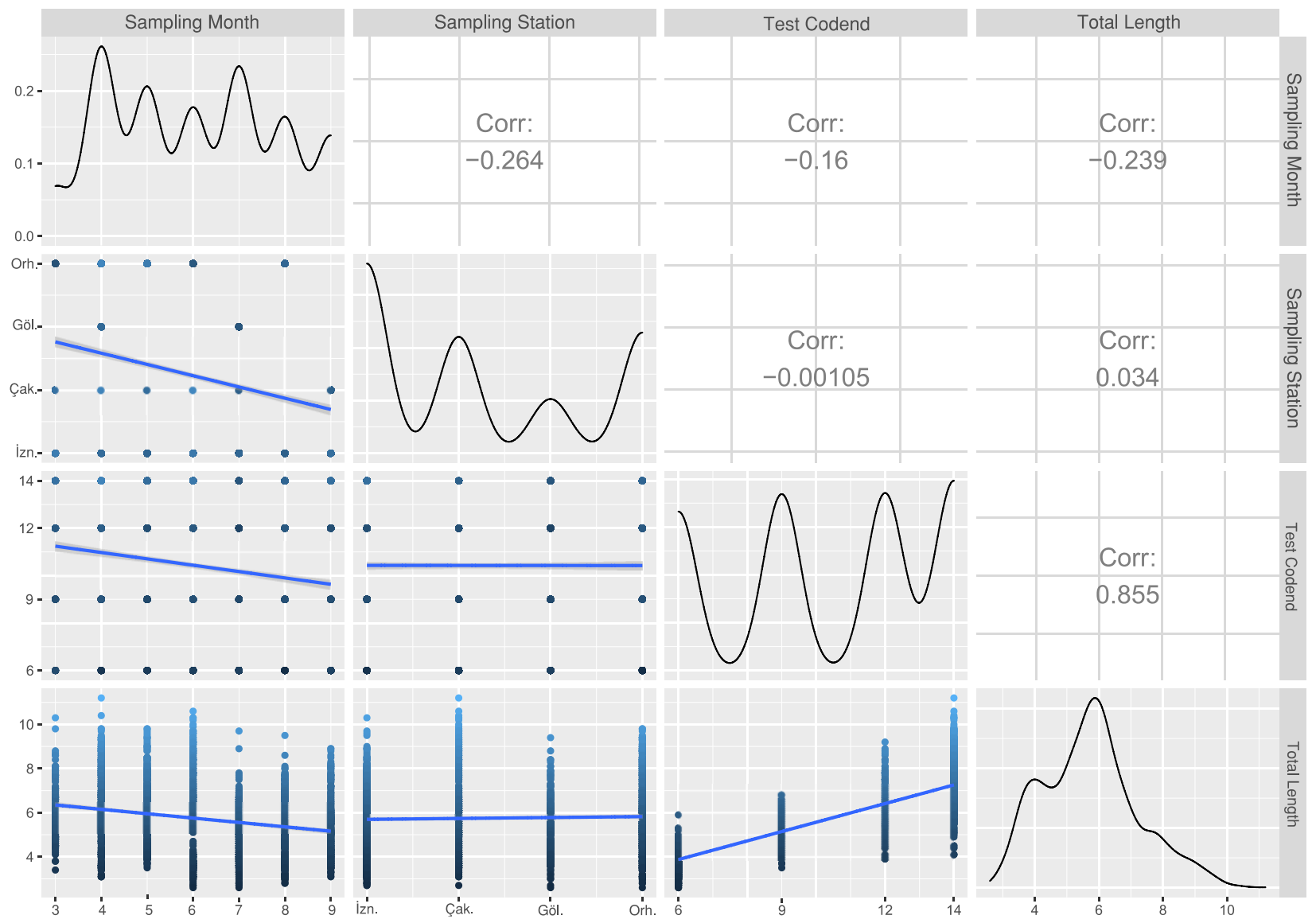

Figure 4. Scatter plot matrix for predictors used GAMs analysis.

Table 3. $K$-S test result of $A$. boyeri captured from different codend

\begin{tabular}{lccccc}
\hline Net 1 & \multicolumn{2}{c}{ Net 2 } & \multicolumn{2}{c}{ Kolmogorov-Smirnov Test } \\
\hline Mesh Size & $\mathrm{N}$ & Mesh Size & $\mathrm{N}$ & D max & Critical Values ( $\alpha=0.05)$ \\
\hline 14 & 824 & 12 & 771 & 0,3288 & 0,0681 \\
14 & 824 & 9 & 767 & 0,3138 & 0,6820 \\
14 & 824 & 6 & 691 & 0,3741 & 0,0702 \\
12 & 771 & 9 & 767 & 0,2805 & 0,0694 \\
12 & 711 & 6 & 771 & 0,4123 & $H_{0}$ Reject \\
9 & 767 & 6 & 691 & 0,3569 & $H_{0}$ Reject \\
\hline
\end{tabular}



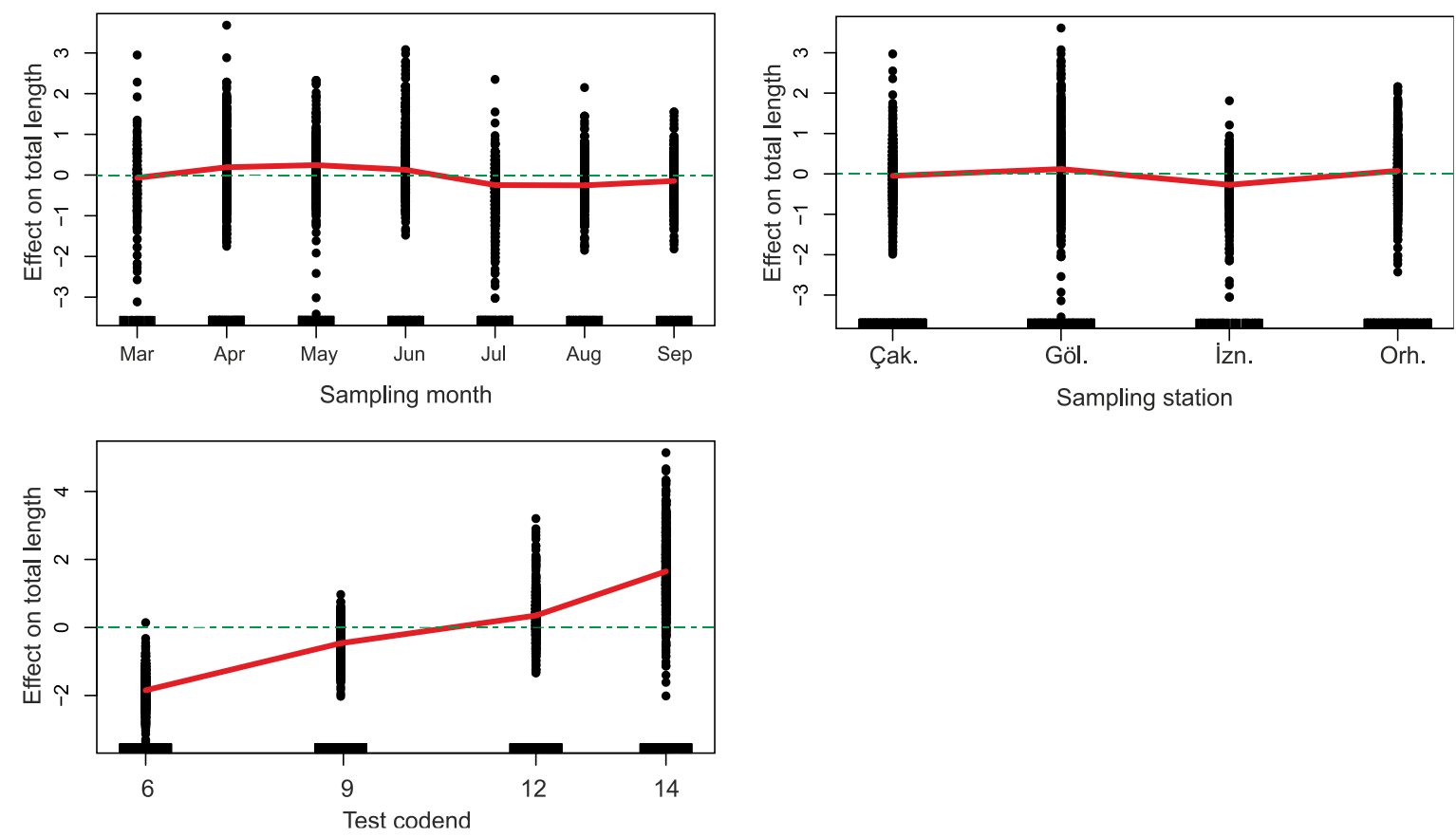

Figure 5. The relationships between total length and other factors from fitted GAMs.

Data by pooling process are given in Table 4 and explain in Figure 3. From the data, statistical differences were found $(P<0.05)$ mean total length of fish that obtained from both codend and cover of beach seine.

\section{Selectivity Parameters}

Catch ratio, retrieved from codend were founded as $26.36(\%), 51.76(\%)$ and $77.14(\%)$ for 14,12 and $9 \mathrm{~mm}$ codend respectively (Figure 6). Selectivity parameters of experimental codend's are given Table $5,6,7$. L50 lengths and $S R$ values were determined as $6.71(0.76)$, $5.62(0.59)$ and $4.51(0.35) \mathrm{cm}$ for 14,12 and $9 \mathrm{~mm}$ codends, respectively. Statistical differences were found between $L_{50}$ values of the codends $(P<0.05)$. There are no statistical differences between 12 and $14 \mathrm{~mm}$ codends $S R$ values. However, statistical difference was estimated $(P<0.05)$. When comparing to this value with $9 \mathrm{~mm}$, Selectivity curves and normalized frequency plots are given Figure 4. Estimated $L_{50}$ and $S R$ values are given Figure 5 , in addition length at first maturity of $A$. boyeri reported by Anonymus (2015) are shown same graph for male $(4.52 \mathrm{~cm})$ and female $(4.96 \mathrm{~cm})$ separately.

Estimated $L_{50}$ vs $S R$ values and its standard errors shown in Figure 7. Codend with $12 \mathrm{~mm}$ mesh size is suitable for sustainable $A$. boyeri fishery with beach seine net by length at first maturity size of species.

\section{Discussion}

It is first time presented beach seine selectivity for A. boyeri in the study. 13 successfully hauls were utilized for estimating selectivity parameters. However, totally 48 fishing operations were performed. Due to the not obtained, 35 hauls not including the data for estimated selectivity parameters. A. boyeri schools migrating to deeper water in conjunction with decreasing the water temperature in Fall and Winter seasons, so it could not be caught in these months except September.

Most of samples were obtained in April, while least were in March (Table 1). Production shown decreasing trend towards fall season and there are no individuals in late fall and winter season. This circumstance associated with bio-ecological properties of $A$. boyeri. In general, as a species originated from mild climate marine system, $A$. boyeri migrate to deeper areas for protect itself in cold water temperature. This case directly connected with water temperature by Işıklıkaya (2017), although $A$. boyeri is a species that can be adapted very hard conditions, if water temperature is decreasing below $8^{\circ} \mathrm{C}$ feeding of species comes to halt; moreover, water temperature below the $4^{\circ} \mathrm{C}$ is cause deadly effect on $A$. boyeri. Whereupon it is seeing that $A$. boyeri migrate to marine from freshwater system if there is a connection between both of them; otherwise flocks are clustering relatively hotter deeper locations of lake's in winter. It is impossible catching of this fish with beach seine in this circumstance.

Naturally mean total length were increase in keeping with mesh size increasing used in codend (Figure 5). Similar observations are seen $L_{50}$ values (Figure 7). These results shown similarity to other selectivity studies based on logistic model (Bolat, Demirci \& Mazlum, 2010; Dereli et al., 2016; ilkyaz et al, 2017; Nguyen \& Larsen, 2013; Reeves et al., 1992). Risk of selectivity modal used in this study was pilling probability of the school front of codend panel. In this situation, smaller fish would not pass from one compartment to others. $A$. boyeri scholl has a specifically spiral circle movement and this habit was 
Table 4. Pooled data and mean length

\begin{tabular}{|c|c|c|c|c|c|c|c|}
\hline \multirow{3}{*}{ Codend Mesh Size (mm) } & \multicolumn{6}{|c|}{ Total Length $(\mathrm{cm})$} & \multirow{3}{*}{$P$} \\
\hline & \multicolumn{2}{|c|}{ Codend } & & & \multicolumn{2}{|c|}{ Cover } & \\
\hline & Pooled Codend & $N$ & Mean $\pm S E$ & Pooled Codend & $N$ & Mean $\pm S E$ & \\
\hline 14 & 14 & 824 & $7.43 \pm 0.04^{c}$ & $12+9+6$ & 2229 & $5.12 \pm 0.02^{c}$ & 0.000 \\
\hline 12 & $14+12$ & 1595 & $6.79 \pm 0.03^{b}$ & $9+6$ & 1458 & $4.61 \pm 0.02^{b}$ & 0.000 \\
\hline 9 & $14+12+9$ & 2362 & $6.30 \pm 0.02^{\mathrm{a}}$ & 6 & 691 & $3.87 \pm 0.02^{\mathrm{a}}$ & 0.000 \\
\hline
\end{tabular}

Different letters within columns show significant differences between codends $(P<0.05)$.

Table 5. Selectivity parameters of $A$. boyeri for codend with $14 \mathrm{~mm}$

\begin{tabular}{|c|c|c|c|c|c|c|c|c|c|c|c|c|c|c|c|}
\hline Parameters & Op1 & Op2 & Op3 & Op4 & Op5 & Op6 & Op7 & Op8 & Op9 & Op10 & Op11 & Op12 & Op13 & $P$ & $F$ \\
\hline$L_{50}$ & 7.03 & 6.78 & 6.56 & 6.77 & 7.21 & 6.85 & 7.01 & 7.82 & 6.27 & 6.18 & 6.65 & 6.53 & 6.48 & 6.69 & 6.71 \\
\hline$L_{50}(S E)$ & 1.05 & 1.00 & 1.00 & 1.04 & 1.03 & 1.00 & 1.00 & 1.00 & 1.00 & 1.00 & 1.00 & 1.00 & 1.00 & 1.00 & 0.11 \\
\hline$L_{50}$ & $-6.36 /$ & $-5.98 /$ & $-6.17 /$ & $-6.50 /$ & $-5.94 /$ & $-5.87 /$ & $-5.74 /$ & $-5.00 /$ & $-6.50 /$ & $-6.56 /$ & $-6.13 /$ & $-6.20 /$ & $-6.24 /$ & $-6.02 /$ & 6.47/ \\
\hline$(\% 95 C l)$ & 20.42 & 19.54 & 19.29 & 20.04 & 20.37 & 19.58 & 19.76 & 20.65 & 19.05 & 18.93 & 19.45 & 19.27 & 19.22 & 19.41 & 6.95 \\
\hline$S R$ & 1.75 & 0.69 & 0.75 & 0.58 & 1.49 & 0.48 & 0.69 & 0.75 & 1.05 & 0.97 & 1.12 & 0.45 & 0.49 & 1.00 & 0.76 \\
\hline$S R(S E)$ & 1.09 & 1.00 & 1.00 & 1.02 & 1.05 & 1.00 & 1.00 & 1.01 & 1.01 & 1.00 & 1.01 & 1.00 & 1.00 & 1.00 & 0.07 \\
\hline$S R$ & $-12.12 /$ & $-12.08 /$ & $-12.00 /$ & $-12.44 /$ & $-11.96 /$ & $-12.25 /$ & $-12.08 /$ & $-12.12 /$ & $-11.83 /$ & $-11.82 /$ & $-11.79 /$ & $-12.29 /$ & $-12.25 /$ & $-11.72 /$ & $0.61 /$ \\
\hline$(\% 95 C l)$ & 15.63 & 13.46 & 13.50 & 13.61 & 14.95 & 13.21 & 13.47 & 13.63 & 13.94 & 13.77 & 14.03 & 13.20 & 13.24 & 13.74 & 0.92 \\
\hline$R_{1}$ & 1.111 & 1.009 & 1.004 & 1.091 & 1.072 & 1.003 & 1.008 & 1.019 & 1.011 & 1.006 & 1.013 & 1.004 & 1.006 & 1.002 & 0.013 \\
\hline$R_{2}$ & 0.117 & 0.001 & 0.001 & 0.035 & 0.067 & 0.001 & 0.003 & -0.007 & 0.007 & 0.004 & 0.007 & 0.000 & 0.002 & 0.001 & -0.000 \\
\hline$R_{3}$ & 1.193 & 1.010 & 1.007 & 1.051 & 1.122 & 1.004 & 1.011 & 1.027 & 1.028 & 1.014 & 1.033 & 1.006 & 1.006 & 1.004 & 0.005 \\
\hline$a$ & -8.82 & -21.59 & -19.21 & -25.64 & -10.63 & -31.35 & -22.32 & -22.91 & -13.12 & -13.99 & -13.04 & -31.88 & -29.05 & -14.56 & -19.39 \\
\hline$b$ & 1.25 & 3.18 & 2.92 & 3.78 & 1.47 & 4.57 & 3.18 & 2.92 & 2.09 & 2.26 & 1.96 & 4.88 & 4.48 & 2.17 & 2.89 \\
\hline Deviance & 8.81 & 6.96 & 4.99 & 1.95 & 15.27 & 1.78 & 13.15 & 3.32 & 2.71 & 10.29 & 3,54 & 3.99 & 5.07 & 44.66 & - \\
\hline$D f$ & 10 & 12 & 12 & 9 & 8 & 12 & 12 & 15 & 7 & 12 & 10 & 10 & 11 & 16 & - \\
\hline \multirow{3}{*}{ Weight } & 48.68 & 397.4 & 267.28 & 266.25 & 40.19 & 333.6 & 134.16 & 330.76 & 93.05 & 143.87 & 123.17 & 129.83 & 177.22 & \multicolumn{2}{|c|}{2485.46} \\
\hline & 100.96 & 118.5 & 293.87 & 28.69 & 112.98 & 305.87 & 202.6 & 137.35 & 152.84 & 248.57 & 164.49 & 149.03 & 241.01 & \multicolumn{2}{|c|}{2256.76} \\
\hline & 149.64 & 515.9 & 561.15 & 294.94 & 153.17 & 639.47 & 336.76 & 468.11 & 245.89 & 392.44 & 287.66 & 278.86 & 418.23 & \multicolumn{2}{|c|}{4742.22} \\
\hline Cod. & 26 & 100 & 100 & 50 & 20 & 100 & 48 & 61 & 50 & 77 & 50 & 50 & 62 & \multicolumn{2}{|c|}{794} \\
\hline Number Cov. & 109 & 99 & 271 & 22 & 113 & 269 & 219 & 103 & 150 & 296 & 150 & 150 & 278 & \multicolumn{2}{|c|}{2229} \\
\hline Tot. & 134 & 199 & 371 & 72 & 133 & 369 & 267 & 164 & 200 & 373 & 200 & 200 & 340 & \multicolumn{2}{|c|}{3023} \\
\hline
\end{tabular}

Table 6. Selectivity parameters of $A$. boyeri for codend with $12 \mathrm{~mm}$

\begin{tabular}{|c|c|c|c|c|c|c|c|c|c|c|c|c|c|c|c|}
\hline Parameters & Op1 & Op2 & Op3 & Op4 & Op5 & Op6 & Op7 & Op8 & Op9 & Op10 & Op11 & Op12 & Op13 & $\mathrm{P}$ & $F$ \\
\hline$L_{50}$ & 5.59 & 5.71 & 5.64 & 4.43 & 5.67 & 5.87 & 5.88 & 6.14 & 5.38 & 5.17 & 5.63 & 5.52 & 5.46 & 5.58 & 5.62 \\
\hline$L_{50}(S E)$ & 1.00 & 1.00 & 1.00 & 1.29 & 1.00 & 1.00 & 1.00 & 1.00 & 1.00 & 1.00 & 1.00 & 1.00 & 1.00 & 1.00 & 0.07 \\
\hline$L_{50}$ & $-7.15 /$ & $-7.01 /$ & $-7.07 /$ & $-12.05 /$ & $-7.09 /$ & $-6.84 /$ & $-6.86 /$ & $-6.61 /$ & $-7.33 /$ & $-7.58 /$ & $-7.10 /$ & $-7.20 /$ & $-7.25 /$ & $-7.11 /$ & $5.48 /$ \\
\hline$(\% 95 \mathrm{Cl})$ & 18.33 & 18.45 & 18.36 & 20.93 & 18.43 & 18.59 & 18.62 & 18.90 & 18.10 & 17.92 & 18.37 & 18.26 & 18.18 & 18.29 & 5.77 \\
\hline$S R$ & 0.73 & 0.49 & 0.66 & 1.33 & 0.80 & 0.60 & 0.81 & 0.51 & 0.31 & 0.79 & 0.62 & 0.55 & 0.42 & 0.74 & 0.59 \\
\hline$S R(S E)$ & 1.00 & 1.00 & 1.00 & 1.32 & 1.00 & 1.00 & 1.00 & 1.01 & 1.00 & 1.00 & 1.00 & 1.00 & 1.00 & 1.00 & 0.04 \\
\hline$S R$ & $-12.08 /$ & $-12.26 /$ & $-12.07 /$ & $-15.55 /$ & $-12.02 /$ & $-12.12 /$ & $-12.00 /$ & $-12.35 /$ & $-12.41 /$ & $-11.98 /$ & $-12.15 /$ & $-12.19 /$ & $-12.26 /$ & $-11.96 /$ & $0.49 /$ \\
\hline$(\% 95 \mathrm{Cl})$ & 13.55 & 13.26 & 13.41 & 18.22 & 13.62 & 13.34 & 13.62 & 13.37 & 13.03 & 13.58 & 13.39 & 13.31 & 13.19 & 13.46 & 0.69 \\
\hline$R_{1}$ & 1.005 & 1.004 & 1.002 & 1.685 & 1.009 & 1.002 & 1.006 & 1.008 & 1.002 & 1.007 & 1.005 & 1.005 & 1.002 & 1.000 & 0.005 \\
\hline$R_{2}$ & 0.000 & -0.000 & -0.000 & -0.640 & 0.001 & -0.000 & -0.002 & -0.000 & -0.000 & -0.000 & -0.001 & -0.000 & 0.000 & -0.000 & 0.000 \\
\hline$R_{3}$ & 1.017 & 1.009 & 1.006 & 1.766 & 1.018 & 1.005 & 1.016 & 1.024 & 1.003 & 1.012 & 1.010 & 1.007 & 1.003 & 1.000 & 0.002 \\
\hline$a$ & -16.82 & -25.60 & -18.77 & -7.31 & -15.57 & -21.49 & -15.95 & -26.45 & -38.13 & -14.37 & -19.95 & -22.05 & -26.08 & -16.56 & -20.92 \\
\hline$b$ & 3,00 & 4.48 & 3.32 & 1.65 & 2.74 & 3.66 & 2.71 & 4.30 & 7.08 & 2.78 & 3.54 & 3.99 & 4.77 & 2.96 & 3.72 \\
\hline Deviance & 4.60 & 1.39 & 7.38 & 0.37 & 6.48 & 3.37 & 9.90 & 1.04 & 1.29 & 24.38 & 7.46 & 3.88 & 2.66 & 17.27 & - \\
\hline$D f$ & 10 & 12 & 12 & 9 & 8 & 12 & 12 & 14 & 7 & 12 & 10 & 10 & 11 & 16 & - \\
\hline \multirow{3}{*}{ Weight } & 102.72 & 483.4 & 431.51 & 291.71 & 103.66 & 492.92 & 244.63 & 427.11 & 177 & 272.59 & 216.66 & 207.19 & 280.34 & \multicolumn{2}{|c|}{3731.44} \\
\hline & 49.87 & 32.5 & 129.64 & 3.23 & 49.51 & 146.55 & 92.13 & 41 & 68.89 & 119.85 & 71 & 71.67 & 137.89 & \multicolumn{2}{|c|}{1013.73} \\
\hline & 152.59 & 515.9 & 561.15 & 294.94 & 153.17 & 639.47 & 336.76 & 468.11 & 245.89 & 392.44 & 287.66 & 278.86 & 418.23 & \multicolumn{2}{|c|}{4745.17} \\
\hline Cod. & 66 & 147 & 200 & 69 & 61 & 200 & 115 & 96 & 100 & 173 & 100 & 100 & 138 & \multicolumn{2}{|c|}{1565} \\
\hline Number Cov. & 69 & 52 & 171 & 3 & 72 & 169 & 152 & 68 & 100 & 200 & 100 & 100 & 202 & \multicolumn{2}{|c|}{1458} \\
\hline Tot. & 135 & 199 & 371 & 72 & 133 & 369 & 267 & 164 & 200 & 373 & 200 & 200 & 340 & \multicolumn{2}{|c|}{3023} \\
\hline
\end{tabular}



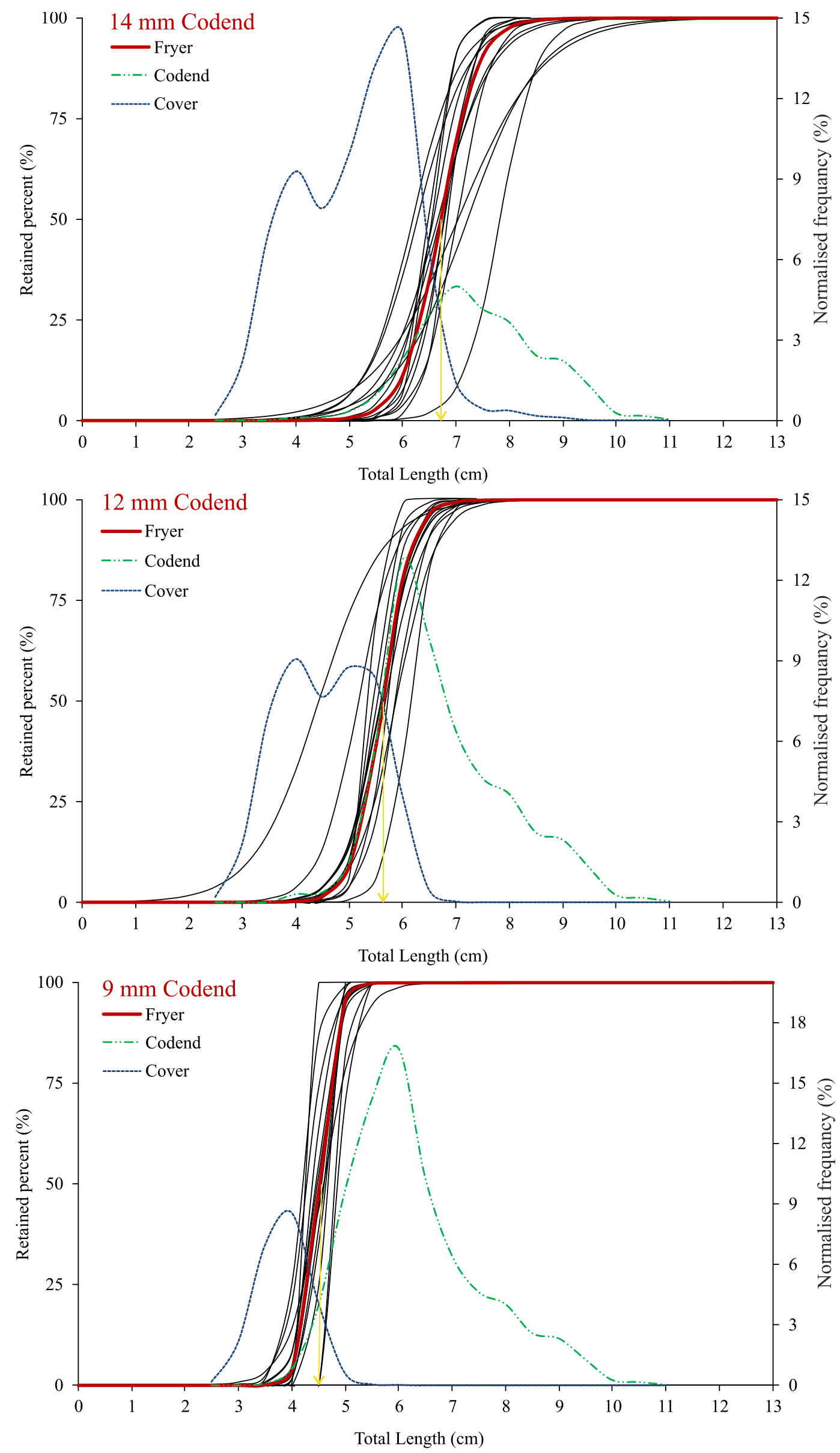

Figure 6. Selection curves and length distribution of $A$. boyeri for different codend. 
Table 7. Selectivity parameters of $A$. boyeri for codend with $9 \mathrm{~mm}$

\begin{tabular}{|c|c|c|c|c|c|c|c|c|c|c|c|c|c|c|c|c|}
\hline \multicolumn{2}{|c|}{ Parameters } & Op1 & Op2 & Op3 & Op4 & Op5 & Op6 & Op7 & Op8 & Op9 & Op10 & Op11 & Op12 & Op13 & $\mathrm{P}$ & $F$ \\
\hline \multicolumn{2}{|l|}{$L_{50}$} & 4.58 & 4.21 & 4.56 & $N A$ & 4.42 & 4.93 & 4.55 & 4.96 & 4.59 & 4.18 & 4.28 & 4.53 & 4.46 & 4.48 & 4.51 \\
\hline \multicolumn{2}{|l|}{$L_{50}(S E)$} & 1.00 & 1.05 & 1.00 & $N A$ & 1.00 & 1.00 & 1.03 & 1.52 & 1.00 & 1.00 & 1.00 & 1.00 & 1.00 & 1.00 & 0.06 \\
\hline \multirow{2}{*}{\multicolumn{2}{|c|}{$\begin{array}{l}L_{50} \\
(\% 95 \mathrm{Cl})\end{array}$}} & $-8.22 /$ & $-9.22 /$ & $-8.16 /$ & & $-12.44 /$ & $-7.86 /$ & $-8.59 /$ & $-62.89 /$ & $-8.13 /$ & $-8.53 /$ & $-8.46 /$ & $-8.19 /$ & $-8.24 /$ & $-8.22 /$ & $4.38 /$ \\
\hline & & 17.38 & 17.64 & 17.28 & & 13.07 & 17.74 & 17.69 & 72.82 & 17.32 & 16.90 & 17.02 & 17.26 & 17.18 & 17.19 & 4.64 \\
\hline \multicolumn{2}{|l|}{$S R$} & 0.71 & 0.10 & 0.30 & $N A$ & 0.31 & 0.10 & 0.10 & 0.10 & 0.32 & 0.37 & 0.46 & 0.33 & 0.42 & 0.44 & 0.35 \\
\hline \multicolumn{2}{|l|}{$S R(S E)$} & 1.01 & 1.01 & 1.00 & & 1.00 & 1.01 & 1.12 & 1.52 & 1.00 & 1.00 & 1.00 & 1.00 & 1.00 & 1.00 & 0.02 \\
\hline \multicolumn{2}{|l|}{$S R$} & $-12.13 /$ & $-12.78 /$ & $-12.42 /$ & & $-12.44 /$ & $-12.85 /$ & $-14.22 /$ & $-19.32 /$ & $-12.40 /$ & $-12.34 /$ & '-12.29/ & $-12.40 /$ & $-12.30 /$ & $-12.26 /$ & $0.30 /$ \\
\hline \multicolumn{2}{|l|}{$(\% 95 \mathrm{Cl})$} & 13.57 & 12.98 & 13.03 & & 13.07 & 13.05 & 14.42 & 19.52 & 13.05 & 13.09 & 13.23 & 13.08 & 13.15 & 13.15 & 0.40 \\
\hline \multicolumn{2}{|l|}{$R_{1}$} & 1.015 & 1.117 & 1.002 & $N A$ & 1.004 & 1.015 & 1.070 & 28.52 & 1.003 & 1.001 & 1.006 & 1.003 & 1.002 & 1.000 & 0.0037 \\
\hline \multicolumn{2}{|l|}{$R_{2}$} & -0.011 & -0.010 & -0.000 & $N A$ & -0.001 & -0.024 & 0.135 & -0.489 & -0.000 & 0.000 & 0.000 & -0.000 & -0.000 & -0.000 & -0.0003 \\
\hline \multicolumn{2}{|l|}{$R_{3}$} & 1.023 & 1.028 & 1.003 & $N A$ & 1.008 & 1.040 & 1.271 & 2.336 & 1.003 & 1.002 & 1.009 & 1.005 & 1.003 & 1.000 & 0.0005 \\
\hline \multicolumn{2}{|l|}{$a$} & -14.17 & -92.50 & -33.39 & $N A$ & -31.32 & -108.3 & -99.97 & -108.9 & -31.51 & -24.82 & -20.44 & -30.16 & -23.33 & -22.37 & -28.31 \\
\hline \multicolumn{2}{|c|}{$b$} & 3.09 & 21.97 & 7.32 & $N A$ & 7.08 & 21.97 & 21.97 & 21.97 & 6.86 & 5.93 & 4.77 & 6.65 & 5.23 & 4.99 & 6.27 \\
\hline \multicolumn{2}{|c|}{ Deviance } & 2.29 & 0.04 & 0.75 & $N A$ & 1.16 & 0.01 & 0.00 & 0.00 & 6.09 & 2.73 & 6.42 & 1.71 & 3.56 & 4.41 & - \\
\hline \multirow[t]{2}{*}{$D f}$. & & 10 & 12 & 12 & $N A$ & 8 & 12 & 12 & 14 & 7 & 12 & 10 & 10 & 11 & 16 & - \\
\hline & Cod. & 141.95 & 514.28 & 538.11 & 294.94 & 145.33 & 607.24 & 306.82 & 451.56 & 221.95 & 356.38 & 268.61 & 256.29 & 373.89 & 447 & 77.35 \\
\hline \multirow[t]{3}{*}{ Weight } & Cov. & 10.64 & 1.62 & 23.04 & & 7.84 & 32.23 & 29.94 & 16.55 & 23.94 & 36.06 & 19.05 & 22.57 & 44.34 & & 7.82 \\
\hline & Tot. & 152.59 & 515.9 & 561.15 & 294.94 & 153.17 & 639.47 & 336.76 & 468.11 & 245.89 & 392.44 & 287.66 & 278.86 & 418.23 & 474 & 5.17 \\
\hline & Cod. & 115 & 192 & 300 & 72 & 111 & 300 & 164 & 115 & 150 & 273 & 150 & 150 & 240 & & 332 \\
\hline \multirow{2}{*}{ Number } & Cov. & 20 & 7 & 71 & 0 & 22 & 69 & 103 & 49 & 50 & 100 & 50 & 50 & 100 & & 91 \\
\hline & Tot. & 135 & 199 & 371 & 72 & 133 & 369 & 267 & 164 & 200 & 373 & 200 & 200 & 340 & & 023 \\
\hline
\end{tabular}

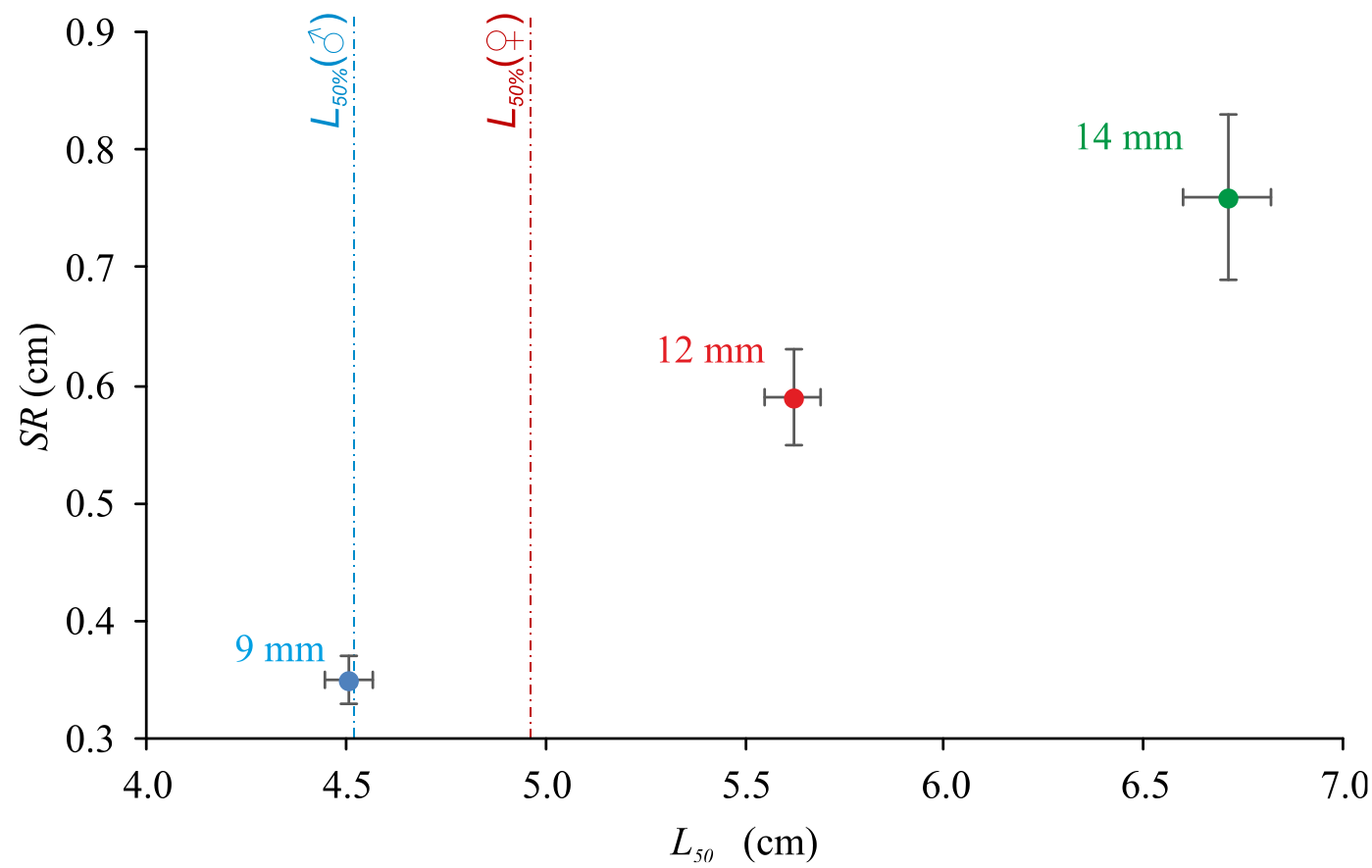

Figure 7. Estimated $L_{50}$ VS $S R$ values and error bars.

going on in seine compartment. The fish which would not pass other codend no waited front of the compartment, they continued circle movement. So, we could not see pilling observation in front of codend mesh. The beach seine compartments used in trial was divided of catch by lengths, this idea supported as scientifically with both mean total length differences and length frequency distribution differences that is revealed by $K-S$ test result (Table 3 ). This result show that applied trial modal has given successfully for selectivity analysis. Cover codend is specified as a properly method for measured selectivity by Pope, Margetts, Hamley and Akyüz (1975) due to no effect escaped all length group of fish from codend. As a disadvantage masking effect expressed by Tosunoğlu and Tokaç (1997) that arising from reposing of cover on codend mesh may be cause preventing effect of fish escaping.

Any masking effect was not observed between codend panels in this study in that each codend panel had $2.5 \mathrm{~m}$ free field front of compartment. Occurred hauling with manpower and very slowly positive 
effected on this circumstance. Other beach seine selectivity study in which used covered codend method was carried out by Tosunoğlu (2003) in Aegean shore of Turkey. The study was conducted based on classically one codend ( $36 \mathrm{~mm}$ ) and one cover net model $(24 \mathrm{~mm})$. Similar study was carried out by (Tesfaye et al., 2016) in Lake Koka (Ethiopia).

Increasing water temperature parallelly increase food web in environment and fish metabolism speed. Therefore, fish condition is also increase. Highest fish condition was founded in August as 0.738 , while lowest was 0.584 in March (Table 1). This circumstance caused on seasonal length frequency differences. Highest condition was reported by Lorenzoni et al. (2015) (Lake Trasimeno, Italy) and Gençoğlu and Ekmekçi (2016) (Hirfanlı Dam Lake, Turkey) for A. boyeri in July.

It was found that there are differences between mean total length of stations $(P<0.05)$ which caught by hauling same time. This result might be stem from the ecological properties food abundance of fishing areas. Also, this finding supports to the assumption that flocks length frequency may be different from each other. Iznik area is shallower than other locations for this reason water temperature is little higher, so it has relatively rich plankton abundance and this cause to positive effect on feeding regime of fish. In addition, many streams (such as, İtimat, Göksu, Karasu, Kavaklık, Sölöz and Kıran Stream) entered in İnik lake, they are also direct and indirectly positive effected on water quality and lake productivity as well.

It is clearly revealed that multimesh codend selectivity model approach is very useful for presented mean lengths differences of fish at same time in different stations. If study had conducted classically one codend - one cover mesh approach, same codend's $L_{50}$ values would be founded different from each other, based on probability of surrounded stocks have different length distribution. In this context, it is thought that selectivity method used in the study can be utilized similar other selectivity studies, if there are no any masking effect front of the codend.

$14 \mathrm{~mm}$ codend produce higher $19 \%$ and $49 \% L_{50}$ values than $12 \mathrm{~mm}$ and $9 \mathrm{~mm}$ codend. $9 \mathrm{~mm}$ codend mesh size is commonly use in $A$. boyeri fishery in Iznik Lake, $L_{50}$ values of $9 \mathrm{~mm}$ codend $(5.62 \mathrm{~cm})$ lower from first maturity length $(4.52 \mathrm{~cm}$ for male and $4.96 \mathrm{~cm}$ for female) given by Anonymous (2015). Rodríguez-Climent et al. (2012) researched gillnet selectivity for A. boyeri using SELECT method in Ebro Delta (North-East of Spain) lagoon. Authors reported catch of gillnet with $10 \mathrm{~mm}$ mesh size were mostly below the first maturity length $(5.22 \mathrm{~cm})$. But, almost all of the caught fish lengths were above first maturity, when mesh size was increased to $12.5 \mathrm{~mm}$. Although used selectivity method is different result are shown similarity with us.

When this species, which is defined as a species with invasive potential by Ekmekçi et al. (2013) and which cannot be completely removed from the Iznik Lake, is considered in terms of its contributions both to the fishermen and the national economy, it is believed that it would be more advantageous to maintain its stock bio-economically. The most important method to prevent overpopulation of this species in the Iznik Lake is the fishing pressure since it does not have an effective predator. In summary, fishermen should continue to catch this species in order to prevent excessive reproduction of the species in the lake. In order the fishermen to continue fishing, the members of the stock should grow in a certain range of length $(5-6 \mathrm{~cm})$ in terms of marketability.

There are very few studies on the selectivity of beach seine, which is widely used in small scale fisheries in many regions of the world. In addition to the selectivity of these sets, which are generally equipped with fine-meshed nets, there is a need for studies on their impact on the coastal ecosystem. In particular, it is necessary to determine the non-target ratios of other species on larvae and juvenile. In some areas, fishermen remove the structures such as stones, rocks, etc. from the environment in order to avoid the lead to be stuck during hauling; and this causes the destruction in the natural structure of the ground. In line with the current findings, it is believed that the method we used to reveal the selectivity of more than one codend simultaneously through the logistic model was very successful and could be used easily in studies of similar cases.

It is thought that logistic model is successfully for estimating multimesh codend selectivity. So current model can be adapted and use for similar other locations and different fish species. One important point that should not forget is selectivity not only related with net mesh size but also fish behavior (Tokai et al., 1995; Wileman et al., 1996; Fonseca et al., 2005). Hence, determining of beach seine codend selectivity with classical method (one codend - one cover) may be helpful for comparing of gained result in study.

\section{Acknowledgement}

This study was supported by Republic of Turkey, Ministry of Agriculture and Forest, General Directorate of Agricultural Research and Policies [grant number: TAGEM/HAYSÜD/2015/A11/P-02/1] and which was presented in $1^{\text {st }}$ International Symposium on Limnology and Freshwater Fisheries.

\section{References}

Akyol, O. (2003). Retained and Trash Fish Catches of BeachSeining in the Aegean Coast of Turkey. Turkish Journal of Veterinary and Animal Sciences, 27(5), 1111-1117. https://www.semanticscholar.org/paper/Retained-andTrash-Fish-Catches-of-Beach-Seining-inAkyol/ecc89e08b575a93e169a946704d40fca291702b6

Anonymous, (2015). Estimating of big scale sand-smelt (Atherina boyeri Risso, 1810) stock and determining of selectivity properties of gear used it's fishing in iznik Lake [in Turkish]. Unpublished project final report. Republic of 
Turkey, Ministry of Agriculture and Forrest, General Directory of Agricultural Research and Policy. 100p.

Battalgil, F. (1941). Les poissons des Eaux Dources de la Turquie, Revue de la Faculté des Sciences de l'Université d'Istanbul, Série B, Sciences Naturelles, Seri B, 6, 170186.

Bolat, Y., Demirci, A. \& Mazlum, Y. (2010). Size Selectivity of Traps (Fyke-Nets) of Different Mesh Size on The NarrowClawed Crayfish, Astacus leptodactylus (ESCHSCHOLTZ, 1823) (Decapoda, Astacidae) İ Eğirdir Lake, Turkey. Crustaceana, 8(11), 1349-1361.

https://brill.com/view/journals/cr/83/11/articlep1349_6.xml?language $=e n$

Brčić, J., Herrmann, B., Mašanović, M., Baranović, M., Šifner, S. K., \& Škeljo, F. (2018). Size selection of Nephrops norvegicus (L.) in commercial creel fishery in the Mediterranean Sea. Fisheries Research, 200, 25-32. https://doi.org/10.1016/j.fishres.2017.12.006

Broadhurst, M.K., Millar, R.B., Brand, C.P. \& Uhlmann, S.S. (2008). Mortality of discards from southeastern Australian beach seines and gillnets. Diseases of Aquatic Organisms, 80(1), 51-61. http://dx.doi.org/10.3354/dao01904

Cabral, H., Duque, J. \& Costa, M. J. (2003). Discards of the beach seine fishery in the central coast of Portugal. Fisheries Research, 63(1), 63-71. http://dx.doi.org/10.1016/s0165-7836(03)00004-3

Ceribasi, G. (2018). Analysis of meteorological and hydrological data of Iznik lake basin by using innovative sen method. Journal of Environmental Protection and Ecology, 19(1), 15-24.

ConStat (1995). CC selectivity. Granspaettevej 10, DK9800 Hjjlarring, Denmark.

Dereli, H., Aydin, C., Belli, M., Kebapçioğlu, T., Akpinar, I.Ö. \& Şen, Y. (2016). Selectivity of commercial and experimental codends for the demersal trawl fishery of the deep-water rose shrimp, parapenaeus longirostris (Lucas, 1846), In the aegean sea. Crustaceana, 89(4), 477-493. http://dx.doi.org/10.1163/15685403-00003532

Deval, M. C., Özgen, G., \& Özbilgin, H. (2016). Selectivity of 50 $\mathrm{mm}$ T0 and T90 codends for commercial shrimp species in the Turkish deepwater trawl fishery, Eastern Mediterranean. 32(6), 1041-1057.

http://dx.doi.org/10.1111/jai.13128

Dogan, K. (2009). Socio-economic analysis of sand smelt fishermen fishing boat owner of Iznik lake (Bursa/Turkey) [in Turkish]. Journal of FisheriesSciences.com, 3(1), 58.

https://www.cabdirect.org/cabdirect/abstract/2009303 9850

Ekmekçi, F.G., Kırankaya, Ş.G., Gençoğlu, L. \& Yoğurtçuoğlu, B. (2013). Present status of invasive fishes in inland waters of Turkey and assessment of the effects of invasion [in Turkish]. Journal of Fisheries \& Aquatic Sciences, 28, 105140.

El-Mor, M., El-Etreby, S. \& Sapota, M. R. (2002). Species composition and size structure of beach seine by-catches in port-said fishing Harbour, Egypt. Oceanological Studies, 31(3-4), 31-43.

Fonseca, P., Campos, A., Mendes, B. \& Larsen, R.B. (2005). Potential use of a Nordmore grid for by-catch reduction in a Portuguese bottom-trawl multispecies fishery. Fish. Res, 73, 49-66.

http://dx.doi.org/10.1016/j.fishres.2005.01.005
Fryer, R. J. (1991). A model of between-haul variation in selectivity. ICES Journal of Marine Science, 48(3), 281 290. http://dx.doi.org/10.1093/icesjms/48.3.281.

Fryer, R. J., \& Shepherd, J. G. (1996). Models of codend size selection. Journal of Northwest Atlantic Fishery Science, 19, 51-58. doi:http://dx.doi.org/10.2960/J.v19.a5

Gençoğlu, L. \& Ekmekçi, F.G. (2016). Growth and reproduction of a marine fish, Atherina boyeri (Risso 1810), in a freshwater ecosystem. Turkish Journal of Zoology, 40(4), 534-542. https://dergipark.org.tr/tr/download/articlefile/436006

Gray, C.A. \& Kennelly, S.J. (2003). Catch characteristics of the commercial beach-seine fisheries in two Australian barrier estuaries. Fisheries Research, 63(3), 405-422. http://dx.doi.org/10.1016/S0165-7836(03)00076-6

Hutchings, K. \& Lamberth, S.J., (2002a). Bycatch in the gillnet and beach-seine fisheries in the Western Cape, South Africa, with implications for management. South African Journal of Marine Science(24), 227-241. http://dx.doi.org/10.2989/025776102784528619

Hutchings, K. \& Lamberth, S.J. (2002b). Catch-and-effort estimates for the gillnet and beach-seine fisheries in the Western Cape, South Africa. South African Journal of Marine Science(24), 205-225. http://dx.doi.org/10.2989/025776102784528529

İlkyaz, A.T., Şensurat, T., Dereli, H. \& Aydın, C. (2017). Codends selectivity for bogue (Boops boops L., 1758) in the eastern mediterranean demersal trawl fishery. Turkish Journal of Fisheries and Aquatic Sciences, 17(4), 673680. http://dx.doi.org/10.4194/1303-2712-v17_4_03

Işıklıkaya, H.E. (2017). Genetic Examination of Atherina boyeri (Sand Smelt) Population Which Lives in İznik Lake(Master Thesis) [in Turkish]. Haliç University, Graduate School of Natural And Applied Sciences. İstanbul, Turkey.

Lorenzoni, M., Giannetto, D., Carosi, A., Dolciami, R., Ghetti, L. \& Pompei, L. (2015). Age, growth and body condition of big-scale sand smelt Atherina boyeri Risso, 1810 inhabiting a freshwater environment: Lake Trasimeno (Italy). Knowledge and Management of Aquatic Ecosystems (416). http://dx.doi.org/10.1051/kmae/2015005

Nguyen, H. P., \& Larsen, R. B. (2013). Effect of codend mesh size increases on the size selectivity of commercial species in a small mesh bottom trawl fishery. Journal of Applied Ichthyology, 29(4), 762-768. http://dx.doi.org/10.1111/jai.12177

Pope, J., Margetts, A., Hamley, J. \& Akyüz, E. (1975). Manual of Methods For Fish Stock Assessment Part III Selectivity Of Fishing Gear Fao Fisheries, Technical Paper No 41 Revision 1.

Reeves, S. A., Armstrong, D. W., Fryer, R. J. \& Coull, K. A.(1992). The effects of mesh size, cod-end extension length and cod-end diameter on the selectivity of scottish trawls and seines. ICES Journal of Marine Science, 49(3), 279288. http://dx.doi.org/10.1093/icesjms/49.3.279

Rodríguez-Climent, S., Alcaraz, C., Caiola, N., Ibáñez, C., Nebra, A., Muñoz-Camarillo, G., Casals, F., Vinloyes, D. \& Sostoa, A. (2012). Gillnet selectivity in the Ebro Delta coastal lagoons and its implication for the fishery management of the sand smelt, Atherina boyeri (Actinopterygii: Atherinidae). Estuarine, Coastal and Shelf Science, 114, 41-49.

http://dx.doi.org/10.1016/j.ecss.2011.09.008

RStudio Team (2019). RStudio: Integrated Development for R. RStudio, Inc., Boston, MA URL http://www.rstudio.com/. 
Sala, A., Brčić, J., Herrmann, B., Lucchetti, A., \& Virgili, M. (2016). Assessment of size selectivity in hydraulic clam dredge fisheries. Canadian Journal of Fisheries and Aquatic Sciences, 74(3), 339-348. http://dx.doi.org/10.1139/cjfas-2015-0199

Wood, S. (2019). MGCV: Mixed GAM Computation Vehicle with Automatic Smoothness Estimation. R package version, 1.8-31. https://cran.rproject.org/web/packages/mgcv/index.html

Tesfaye, G., Wolff, M. \& Taylor, M. (2016). Gear selectivity of fishery target resources in Lake Koka, Ethiopia: evaluation and management implications. Hydrobiologia, 765(1), 277-295. http://dx.doi.org/10.1007/s10750-015-2420-0

Tokai, T., S. Omoto, R. Sato \& K. Matuda, (1995). A method of determining selectivity curve of separator grid. Fish. Res., 27: 51-60. http://dx.doi.org/10.1016/01657836(95)00471-8

Tosunoğlu, Z. (2003). Experiments on the Cod-end Selectivity of Beach Seine Nets on the Turkish Coast of the Aegean Sea. Turkish Journal of Veterinary and Animal Sciences, 27(5), 1049-1055.
Tosunoğlu, Z. \& Tokaç, A. (1997). Sürütme Ağlarının Seçiciliğini Ölçmede Kullanılan Yöntemler. Ege Üniversitesi Su Ürünleri Dergisi, 14(1-2), 219-237.

TUIK, (2019). Turkish Statistical Institute (Fishery Statistic). Retrieved from http://tuik.gov.tr/PreTablo.do?alt_id=1005

Vanderklift, M.A., Jacoby, C.A. \& Horwitz, P. (1998). Comparison of catches taken in dual-mesh beach seines of two different lengths. Marine and Freshwater Research, 49(6), 513-516. http://dx.doi.org/10.1071/MF97257

Yağci, M. A., \& Ustaoğlu, M. R. (2012). Zooplankton fauna of Lake İznik (Bursa, Turkey). Turkish Journal of Zoology, 36(3), 341-350.

http://journals.tubitak.gov.tr/zoology/issues/zoo-1236-3/zoo-36-3-7-1001-36.pdf

Wileman, D.A., Ferro, R.S.T., Fonteyne, R. \& Millar, R. B. (1996). Manual of Methods of Measuring the Selectivity of Towed Fishing Gears. ICES Cooperative Research Report No. 215,126p. 